\title{
The neutron star in HESS J1731-347: Central compact objects as laboratories to study the equation of state of superdense matter
}

\author{
D. Klochkov ${ }^{1}$, V. Suleimanov ${ }^{1,2}$, G. Pühlhofer ${ }^{1}$, D. G. Yakovlev $^{3}$ A. Santangelo ${ }^{1}$, and K. Werner ${ }^{1}$ \\ ${ }^{1}$ Institut für Astronomie und Astrophysik, Universität Tübingen (IAAT), Sand 1, 72076 Tübingen, Germany \\ e-mail: klochkov@astro.uni-tuebingen.de \\ 2 Kazan (Volga region) Federal University, Kremlevskaya 18, 420008 Kazan, Russia \\ 3 Ioffe Institute, Politekhnicheskaya 26, 194021 St. Petersburg, Russia
}

Received 25 July 2014 / Accepted 1 October 2014

\begin{abstract}
Context. Central compact objects (CCOs) in supernova remnants are isolated thermally emitting neutron stars (NSs). They are most probably characterized by a magnetic field strength that is roughly two orders of magnitude lower than that of most of the radio and accreting pulsars. The thermal emission of CCOs can be modeled to obtain constraints on the physical parameters of the star such as its mass, radius, effective temperature, and chemical composition.

Aims. The CCO in HESS J1731-347 is one of the brightest objects in this class. Starting from 2007, it was observed several times with different X-ray satellites. Here we present our analysis of two new XMM-Newton observations of the source performed in 2013 which increase the total exposure time of the data available for spectral analysis by a factor of about five compared to the analyses presented before.

Methods. We use our numerical spectral models for carbon and hydrogen atmospheres to fit the spectrum of the CCO. From our fits, we derive constraints on the physical parameters of the emitting star such as its mass, radius, distance, and effective temperature. We also use the new data to derive new upper limits on the source pulsations and to confirm the absence of a long-term flux and spectral variability.

Results. The analysis shows that atmosphere models are clearly preferred by the fit over the blackbody spectral function. Under the assumption that the X-ray emission is uniformly produced by the entire star surface (supported by the lack of pulsations), hydrogen atmosphere models lead to uncomfortably large distances of the $\mathrm{CCO}$, above $7-8 \mathrm{kpc}$. On the other hand, the carbon atmosphere model formally excludes distances above 5-6 kpc and is compatible with the source located in the Scutum-Crux ( $\sim 3 \mathrm{kpc})$ or NormaCygnus ( $4.5 \mathrm{kpc})$ Galactic spiral arm. We provide and discuss the corresponding confidence contours in the NS mass-radius plane. The measured effective temperature indicates that the NS is exceptionally hot for the estimated age of $\sim 30$ kyr. We discuss possible cooling scenarios to explain this property, as well as possible additional constraints on the star mass and radius from cooling theory.
\end{abstract}

Key words. stars: neutron - stars: atmospheres

\section{Introduction}

More than a third of all young neutron stars (NSs) associated with supernova remnants (SNRs) are observed as central compact objects (CCOs). These point-like X-ray sources are characterized by thermal X-ray spectra with $k T \sim 0.2-0.5 \mathrm{keV}$ and have so far not been found to emit in other electromagnetic wavebands. The CCOs were first considered as a separate class of isolated NSs after their characterization with the Chandra observatory (Pavlov et al. 2002, 2004). Around a dozen CCOs are known so far including candidates. Pulsations with periods between $\sim 0.1 \mathrm{~s}$ and $\sim 0.4 \mathrm{~s}$ have been detected in three of them (e.g., Gotthelf et al. 2013). Lack of magnetic activity such as non-thermal (magnetospheric) emission and lack of associated pulsar wind nebula, together with the low measured spin-down rates or the corresponding upper limits, lead to relatively small estimated magnetic field strengths of the CCOs, $B \lesssim 10^{10}-10^{11} \mathrm{G}$ (Halpern \& Gotthelf 2010b; Gotthelf et al. 2013). This is roughly two orders of magnitude below the field strengths observed in most of the radio and accreting pulsars and in the small group of nearby X-ray emitting dim isolated neutron stars (XDINS). Thus, CCOs apparently represent a class of young $\left(\$ 10^{3}-10^{4} \mathrm{yr}\right)$, low-magnetized, thermally emitting cooling NSs.

The absence of magnetospheric and accretion phenomena potentially provides an undisturbed view of the stellar surface of CCOs. The geometrical and physical properties of the star can be probed through a modeling of the spectral and timing properties of its X-ray emission. The mass and radius of the compact star which can be estimated from the modeling are directly related to the nature of the superdense matter in its interior. Different theoretical models predict different equations of state (EOS) of superdense nuclear matter and, thus, different massradius relations for NSs which can be compared with observations (e.g., Lattimer \& Prakash 2007).

The CCO XMMUJ173203.3-344518 (to be referred as XMMUJ1732 in the following) was discovered with XMM-Newton in 2007 as a point-like X-ray source roughly at the center of the TeV-emitting SNR HESS J1731-347, also known as G 353.6-0.7 (Acero et al. 2009; Tian et al. 2010; Abramowski et al. 2011). The XMM-Newton observations and the subsequent observations with Suzaku (Bamba et al. 2012) and Chandra have shown that the source exhibits a blackbody-like X-ray spectrum with absorption at low energies characterized by $k T \simeq 0.5 \mathrm{keV}$ 
(typical for a $\mathrm{CCO}$ ) and an absorption column density $n_{\mathrm{H}} \simeq$ $1.5 \times 10^{22} \mathrm{~cm}^{-2}$ (Acero et al. 2009; Halpern \& Gotthelf 2010c; Bamba et al. 2012).

No X-ray pulsations have so far been found in XMMU J1732. Our recent analysis of the XMM-Newton timing observations in 2012 presented in Klochkov et al. (2013), hereafter Paper I, yielded an upper limit of $\sim 10 \%$ on the pulsed fraction of sinusoidal pulsations. Although the limit is not very strong, it can indicate that the X-ray emission is roughly uniformly produced by the entire stellar surface. This assumption was adopted in Paper I to couple the emitting area with that of the compact star. Abramowski et al. (2011) argued that the remnant is most likely located either in the Scutum-Crux arm or in the Norma-Cygnus arm, with the corresponding spiral arm distances of $\sim 3 \mathrm{kpc}$ or $\sim 4.5 \mathrm{kpc}$ following the model by Hou et al. (2009). They also derived a lower limit on the distance of $\sim 3.2 \mathrm{kpc}$ based on the measured X-ray absorption and the ${ }^{12} \mathrm{CO}$ emission in the direction of the remnant. The fit of the CCO spectrum with an absorbed blackbody model under an assumption of a canonical NS radius of $10 \mathrm{~km}$ leads to an unrealistic distance estimate of $\sim 30 \mathrm{kpc}$. A solution to a similar problem in case of the non-pulsating $\mathrm{CCO}$ in Cas A, whose distance is well known, was proposed by Ho \& Heinke (2009) (see, however, Pavlov \& Luna 2009, for an alternative explanation). The usage of a carbon atmosphere spectral model allowed Ho $\&$ Heinke (2009) to reconcile the distance to the source with the radius of a canonical NS. Following a similar approach, the carbon atmosphere models described in Suleimanov et al. (2014) have been applied to the CCO in HESS J1731-347 and allowed the estimated distance to the remnant of $\sim 3-4 \mathrm{kpc}$ to be reconciled with the canonical radius of the neutron star as described in Paper I.

Here we present the analysis of two new observations of XMMU J1732 performed with XMM-Newton in 2013 (PI: G. Pühlhofer) which increase the total exposure time of the observations suitable for an accurate spectral analysis by a factor of about five. The new data put much stronger constraints on the source spectral continuum. We apply our carbon and hydrogen atmosphere models to the new spectra and derive new constraints on the physical parameters of the emitting star such as its mass and radius. We discuss possible cooling scenarios of the NS based on the measured effective temperature and the available age estimate. The cooling theory under certain assumptions allows us to put additional constraints on the star mass and radius. The paper is organized as follows. The available observations and the long term monitoring of the source flux are described in Sect. 2. A new upper limit on the source pulsations is provided in Sect. 3. Spectral analysis is described in Sect. 4. The spectral fits using the carbon and hydrogen atmosphere models are presented in Sects. 4.2 and 4.3, respectively. The cooling history of the NS and the corresponding constraints on the mass and radius of the NS are discussed in Sect. 5.

\section{Observational data and the long-term flux history of XMMU J173203.3-344518}

Since 2007, the CCO in HESS J1731-347 has regularly been observed with the XMM-Newton, Chandra, Suzaku, and Swift satellites. Most of the observations are targeted at the SNR HESS J1731-347. A short log of the available observations is provided in Table 1. In this work, we focus on the analysis of the last two observations performed in 2013 with the XMM-Newton orbital observatory (Jansen et al. 2001). We refer to them as the March observation and the October observation in the following.
Table 1. Summary of observations of XMMU J173203.3-344518.

\begin{tabular}{llll}
\hline \hline Date & $\begin{array}{l}\text { Satellite/ } \\
\text { obs. mode }\end{array}$ & $\begin{array}{l}\text { Exposure } \\
{[\mathrm{ks}]}\end{array}$ & Time res. \\
\hline 2007 Feb. 23 & Suzaku/imaging & 41 & $8 \mathrm{~s}$ \\
2007 Mar. 21 & XMM/imaging & 25 & $70 \mathrm{~ms}$ \\
2008 Apr. 28 & Chandra/imaging & 30 & $3.2 \mathrm{~s}$ \\
2009 Feb. 4 & Swift/imaging & 1.4 & $2.5 \mathrm{~s}$ \\
2009 Mar. 9 & Swift/imaging & 1.4 & $2.5 \mathrm{~s}$ \\
2010 May 18 & Chandra/timing & 40 & $2.85 \mathrm{~ms}$ \\
2012 Mar. 2 & XMM/timing & 24 & $0.03 \mathrm{~ms}$ \\
2013 Mar. 7* & XMM/imaging & 72 & $70 \mathrm{~ms}$ \\
2013 Oct. 6* & XMM/imaging & 61 & $70 \mathrm{~ms}$ \\
\hline
\end{tabular}

Notes. ${ }^{(1)}$ Bamba et al. (2012); (2) Klochkov et al. (2013); ${ }^{(3)}$ Halpern \& Gotthelf (2010a); ${ }^{(*)}$ The new observations analyzed in this work.

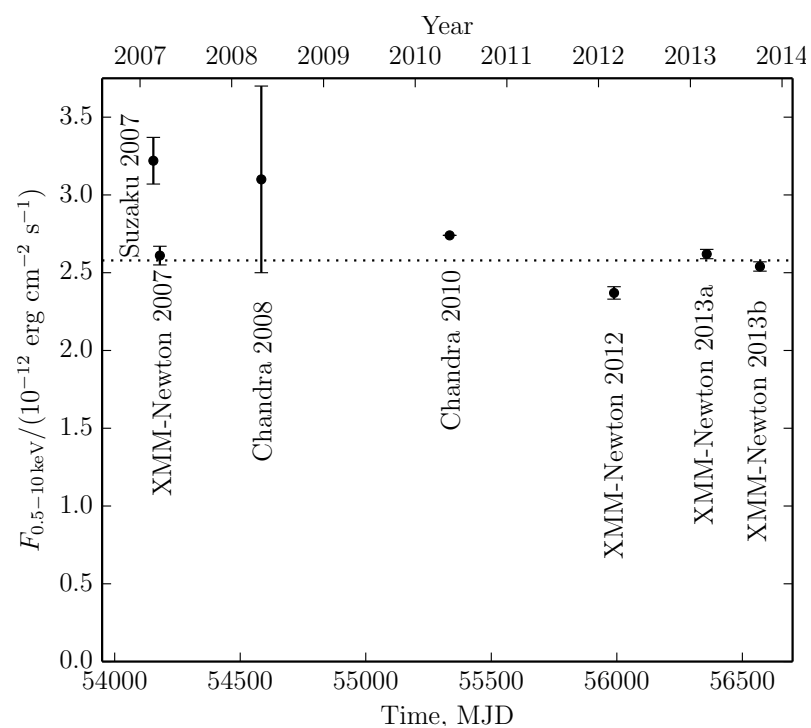

Fig. 1. Absorbed flux measurements of the CCO in HESS J1731-347 in the $0.5-10 \mathrm{keV}$ energy range performed with different instruments. The error bars indicate only statistical errors. For the data point of Chandra 2010 taken from Halpern \& Gotthelf (2010a), no error bar is available. The latest observations analyzed in this work are marked as XMM-Newton 2013a and XMM-Newton 2013b. The horizontal dotted line indicates the averaged flux of the three XMM-Newton observations (2007, 2013a, and 2013b) performed in the same imaging mode.

To combine the data with those taken in the 2007 XMM-Newton observations, we also re-processed the 2007 observations using the latest pipeline version and calibration (see next Section).

The new observations extend the long-term monitoring of the source flux which now covers almost seven years. Figure 1 shows the measured $0.5-10 \mathrm{keV}$ flux (absorbed) as a function of time. The Chandra 2010 data point is taken from Halpern \& Gotthelf (2010a). The error bars indicate only statistical errors. The differences between the flux measurements with different instruments or with the same instrument in different observing modes (imaging/timing) reach $\sim 20 \%$. They can, however, be attributed to imperfect absolute flux calibration of different instruments and modes ${ }^{1}$. The only data points in Fig. 1 which can be compared directly are the XMM-Newton observations in 2007 and 2013: all three observations have been performed in the

1 http://xmm2.esac.esa.int/docs/documents/ CAL-TN-0052.ps.gz 
imaging full frame ${ }^{2}$ mode. The measured fluxes in these observations turn out to be consistent within the statistical errors. The averaged flux over the three observations (indicated by the horizontal dotted line) is $2.58(4) \times 10^{-12} \mathrm{erg} \mathrm{cm}^{-2} \mathrm{~s}^{-1}$. Summarizing the results presented above, we conclude that the available flux measurements are consistent with the source flux being constant over the period covered by the observations.

\section{Upper limits on the pulsed fraction}

The XMM-Newton observations in imaging mode analyzed here permit a search for periodicity below the Nyquist frequency of $f_{\mathrm{Nq}}=1 /\left(2 t_{\mathrm{res}}\right)=6.87 \mathrm{~Hz}$ corresponding to the pulse period of $0.147 \mathrm{~s}\left(t_{\mathrm{res}}=0.0734 \mathrm{~s}\right.$ is the time resolution of EPIC-PN camera in the imaging mode, Strüder et al. 2001). Since the lowest and the highest measured pulse periods of CCOs are $\sim 0.105$ and $0.424 \mathrm{~s}$, there is a good chance to miss pulsations in XMMU J1732 because of the timing resolution limit. We note, however, that the analysis of the $\sim 20 \mathrm{ks}$ timing observations with XMM-Newton presented in Paper I did not reveal any pulsations with the pulsed fraction above $\sim 10 \%$ down to a pulse period of $0.2 \mathrm{~ms}$.

We searched for pulsations in the EPIC-PN data of both $X M M-N e w t o n$ observations performed in 2013. We calculated a Rayleigh periodogram $\left(Z_{1}^{2}\right.$-statistics, e.g., Protheroe 1987, and references therein) for the filtered EPIC-PN event files in the energy range $0.35-5.5 \mathrm{keV}$ extracted with the Science Analysis System (SAS) ${ }^{3}$ version 13.5.0. The standard filtering including the removal of high flaring background intervals has left $\sim 53.2$ and $\sim 58.0 \mathrm{ks}$ of useful source exposure in the March and October observations, respectively. The energy range is a priori chosen as rough compromise between the source-to-background ratio and the total amount of signal counts. No pulsations have been found above $99 \%$ confidence level in either of the two observations. The maximum value of $Z_{1}^{2}$-power is 27.3 for the March and 28.9 for the October observations, respectively, whereas the $99 \%$-threshold values of $Z_{1}^{2}$ in the respective observations are 34.8 and 35.0. The threshold level in each case is calculated taking into account the number of trials which is equal to the number of independent frequencies in the periodogram, $\sim 3.6 \times 10^{5}$ and $\sim 4.0 \times 10^{5}$, in the March and October observations, respectively.

The maximum values of $Z_{1}^{2}$ are converted to upper limits on the pulsed fraction of the source assuming sinusoidal pulsations and using the distributions of $Z_{1}^{2}$ for the case where both the signal and the noise are present in the data (e.g., de Jager et al. 1989). For the March observations, the upper limit on the pulsed fraction of the source signal is $7.3 \%$. For the October observations, it is $8.1 \%$.

\section{Spectral analysis}

We extracted spectra of the CCO in HESS J1731-347 taken with all EPIC cameras (Strüder et al. 2001; Turner et al. 2001) onboard XMM-Newton during the March and October observations 2013. As for the timing analysis above, we used the version 13.5.0 of SAS and the latest calibration files available at the time of the data reduction (February-March 2014). The periods of high flaring background have been identified and excluded

\footnotetext{
2 http://xmm.esac.esa.int/external/xmm_user_support/ documentation/uhb/epicmode.html

3 http://xmm.esac.esa.int/sas/current/documentation/ sas_concise.shtml
}

leading to the reduction of the total exposure by $\sim 25-30 \%$ for the 2013 observations. To combine the new spectra with those obtained with XMM-Newton in 2007 (Table 1, Paper I) we reextracted the 2007 data using the same software and calibration which we used for the 2013 data. In each case, we extracted the source spectrum from a circle with a radius of 40 arcsec centered at the $\mathrm{CCO}$ position and encompassing $~ 90 \%$ of the PSF energy below $\sim 5 \mathrm{keV}$. The background spectra are extracted according to the recommendations of the instrument team, that is from the regions on the same CCD chip having roughly the same RAW Y coordinate as the source for EPIC-PN and from the elliptical annuli with the inner and outer minor semi-axes of $\sim 55^{\prime \prime}$ and $\sim 85^{\prime \prime}$ elongated along the circle of equal off-set radii for EPIC-MOS. The regions with visible diffuse emission and stray light are excluded. The independent spectral analysis of the the three observations did not reveal any variation of the source's spectral shape. The spectra, responses, and background files from the three observations have thus been combined separately for EPIC-PN, EPIC-MOS1, and EPIC-MOS2 cameras using the SAS tool epicspeccombine. All spectral modeling described in the following is performed using the combined spectra.

\subsection{Blackbody versus model atmosphere fits}

We modeled the combined spectra from the three observations with a blackbody function and, alternatively, using the carbon ${ }^{4}$ (Suleimanov et al. 2014) and hydrogen stellar atmosphere models developed by our group. A multiplicative component accounting for the low-energy photo-electric absorption by the interstellar medium was added in each case. The stellar atmosphere models are applied in the same way as described in Paper I. Further details on the stellar atmosphere modeling are provided in the following subsections.

The analyzed spectra allow for the first time the blackbody model to be clearly rejected for this source. The corresponding fit yields a reduced $\chi^{2}=1.33$ for 896 degrees of freedom (d.o.f.). This corresponds to a null-hypothesis probability $\left(P\right.$-value) of $\lesssim 10^{-10}$ indicating a statistically unacceptable fit. The residuals of the fit show systematic wave-like structure (second from top panel in Fig. 2). The best-fit parameters are the following: $n_{\mathrm{H}}=(1.50 \pm 0.02) \times 10^{22}$ atom cm $^{-2}$, $k T=0.489 \pm 0.002 \mathrm{keV}$.

The fits with the carbon and hydrogen stellar atmosphere models yield flat residuals (two bottom panels of Fig. 2) and acceptable fits with a reduced $\chi^{2}=1.07$ for 894 d.o.f. and $P$-values of $\sim 0.1$ in both cases. A more complicated spectral model than a simple blackbody is thus required solely by the shape of the measured spectral continuum.

We verified that the spectral fits described above are not sensitive to the exact choice of the background regions. We thus believe that possible systematic variations of the background over the detector plane close to the source position do not affect our conclusion. We refer to Sect. 4.2 for a discussion of possible systematic effects related to background.

\subsection{Fit with carbon atmosphere models}

Following Paper I, we assumed that the atmosphere characterized by the effective temperature $T$ covers the entire surface of a compact star with mass $M$ and radius $R$ located at a certain

\footnotetext{
4 http://heasarc.gsfc.nasa.gov/xanadu/xspec/models/ carbatm.html
} 


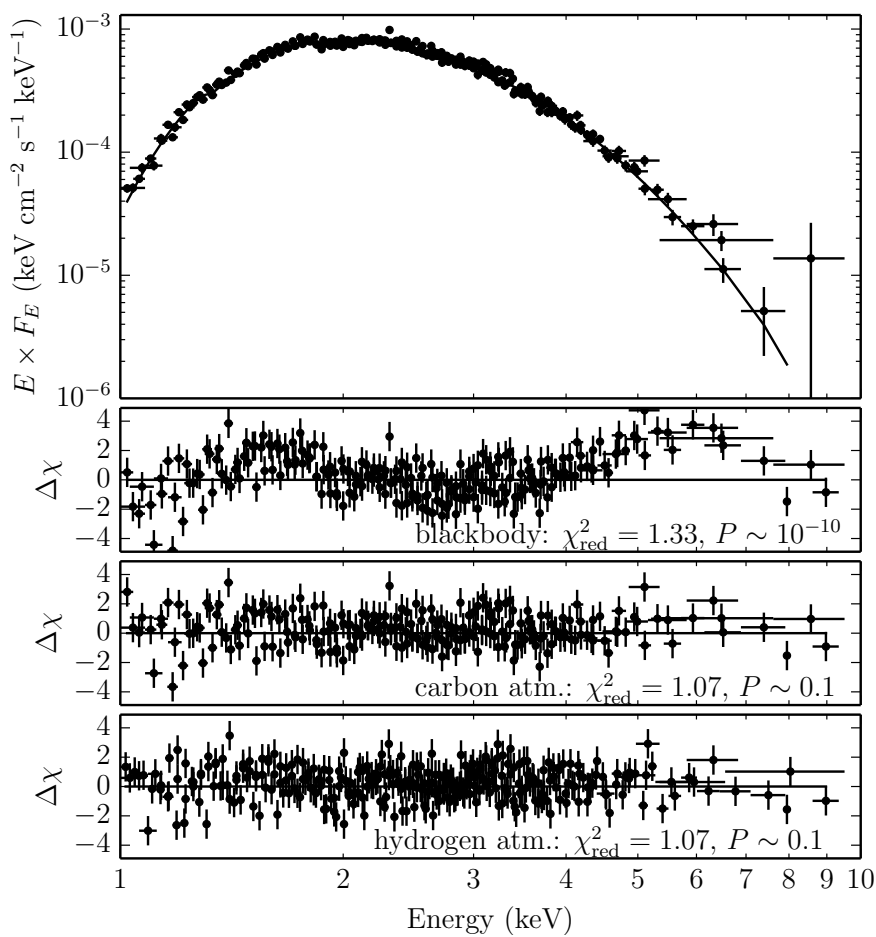

Fig. 2. Combined XMM-Newton spectrum (all EPIC cameras) from the three observations fitted with a carbon atmosphere model (top) and fit residuals for the blackbody, the carbon atmosphere, and the hydrogen atmosphere models (from top to bottom). The null-hypothesis probabilities ( $P$-values) yielded by the $\chi^{2}$-fits are indicated. The blackbody model leads to a statistically unacceptable fit.

distance $d$. Mass and radius are used to calculate the surface gravity and the gravitational redshift affecting the atmosphere model while the distance determines the normalization constant $K$ of the spectrum: $K=1 / d^{2}$. The mass, radius, effective temperature, and the equivalent hydrogen column density $n_{\mathrm{H}}$ which characterizes the photo-electric absorption by the interstellar medium are free fit parameters of the spectral model.

The gravitational redshift $z$ and the surface gravity $g$ affect the shape of the model spectrum in different manners (see Suleimanov et al. 2014, for details). Both values can thus be determined independently from a spectral fit. On the other hand, $M$ and $R$ enter the formulae for $z$ and $g$ in different ways: $1+z=\left[1-2 G M /\left(c^{2} R\right)\right]^{-1 / 2}, g=G M / R^{2}(1+z)$. That is, the curves of equal $z$ in the $M-R$ plane are not parallel to the curves of equal $g$ (see, e.g., Fig. 2 of Suleimanov et al. 2014). A certain pair of $g$ and $z$ values thus uniquely determines a point in the $M-R$ plane. Therefore both mass and radius can in principle be determined independently from a spectral fit as was also demonstrated in Paper I.

As in Paper I, we first used two fixed distances to the source, 3.2 and $4.5 \mathrm{kpc}$, corresponding to the location of the SNR in the Scutum-Crux or Norma arms, respectively (Abramowski et al. 2011). The resulting confidence regions in the $M-R$ plane are presented in Fig. 3. For $d=3.2 \mathrm{kpc}$ we also show the contours obtained with the 2007 observations only (presented in Paper I) for comparison. The dramatic improvement in the statistics (reduction of the contours size) is caused by the increase in the total exposure time by a factor of $\sim$ five. For the best-fit parameters and the associated statistical uncertainties ( $1 \sigma$ c.l. for one parameter of interest) we refer to Table 2 . We note that the confidence levels of the contours in Fig. 3 are plotted for two parameters of interest. The contours corresponding to $68 \%(1 \sigma)$

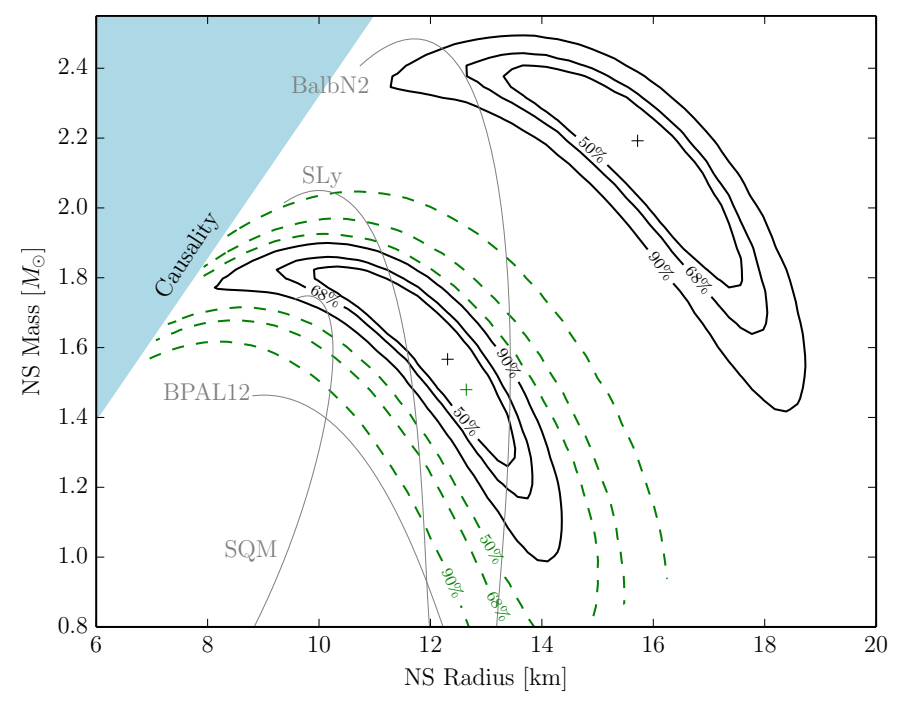

Fig. 3. $\chi^{2}$ confidence regions in the mass-radius plane for the $\mathrm{CCO}$ in HESS J1731-347 obtained with the carbon atmosphere models for the fixed distances of $3.2 \mathrm{kpc}$ (bottom left) and $4.5 \mathrm{kpc}$ (top right). The indicated confidence levels $(50,68$, and $90 \%)$ are for two parameters of interest. The crosses indicate the corresponding $\chi^{2}$-minima. The shaded area in the top left of the plot indicates the region excluded by the requirements of causality. The dashed contours indicate the confidence regions only with the data from the 2007 observations (presented in Klochkov et al. 2013). The thin curves indicate the mass-radius dependencies for some of the commonly used nuclear EOS.

Table 2. Results of the spectral modeling of the $\mathrm{CCO}$ in HESS J1731-347 with carbon atmosphere models.

\begin{tabular}{lll}
\hline \hline$d, \mathrm{kpc}($ fixed) & 3.2 & 4.5 \\
\hline$n_{\mathrm{H}} /\left(10^{22} \mathrm{~cm}^{-2}\right)$ & $2.00 \pm 0.03$ & $1.99_{-0.03}^{+0.02}$ \\
$T, \mathrm{MK}$ & $2.24_{-0.13}^{+0.39}$ & $2.43_{-0.23}^{+0.22}$ \\
$T_{\infty}=T /(1+z), \mathrm{MK}$ & $1.78_{-0.02}^{+0.04}$ & $1.82_{-0.01}^{+0.03}$ \\
$M / M_{\odot}$ & $1.55_{-0.24}^{+0.28}$ & $2.19_{-0.34}^{+0.19}$ \\
$R, \mathrm{~km}$ & $12.4_{-2.2}^{+0.9}$ & $15.7_{-2.0}^{+1.6}$ \\
$\chi_{\text {red }}^{2} /$ d.o.f. & $1.07 / 895$ & $1.07 / 895$ \\
\hline
\end{tabular}

Notes. The indicated uncertainties are at $1 \sigma$ c.l. for one parameter of interest.

confidence level for one parameter of interest (whose projections to the $M$ - and $R$ axes correspond to the uncertainties given in Table 2) would be located slightly within the innermost contours shown in the plot.

The presented contours take into account only statistical uncertainties of the source and background spectra. Additional systematic effects may be related to the spatial variation of the background. Although the background regions are selected close to the source to represent the "local" background (see above), they might still include contributions from the diffuse emission and stray light not directly visible in the image or hidden behind the source's PSF as well as the variations of the instrumental background with respect to that at the source position. To assess possible systematic uncertainties related to these effects, we produced three independent sets of local background regions. Figure 4 presents the confidence contours for the fixed distance of $3.2 \mathrm{kpc}$ (similar to those shown in the bottom left of Fig. 3) obtained with the three sets of background regions. The relative displacements of the contours shown with different colors and line styles reflect the aforementioned systematics. One can see 


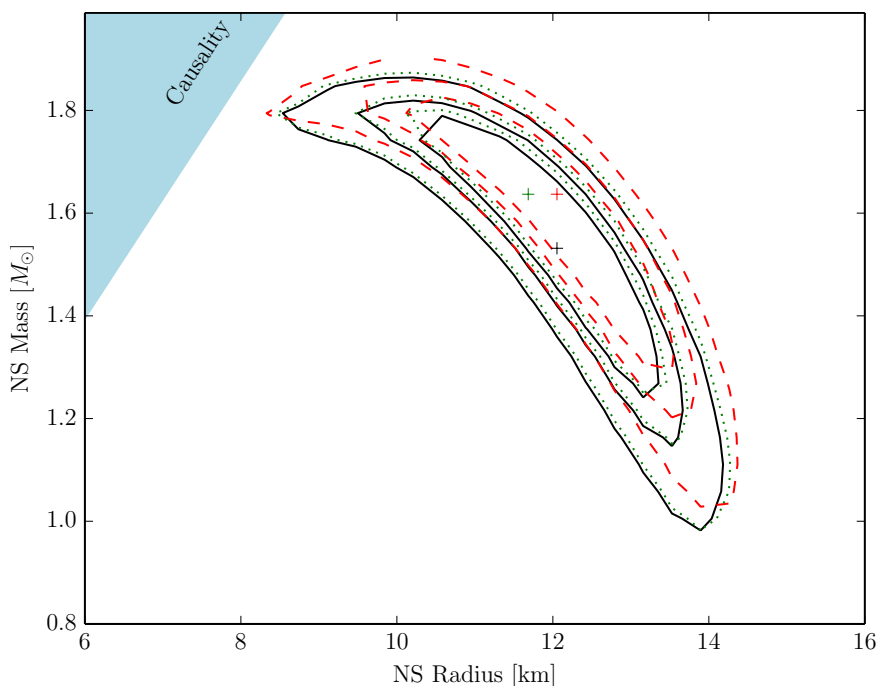

Fig. 4. Confidence regions similar to those shown in Fig. 3 for the fixed distance of $3.2 \mathrm{kpc}$. The different colors and line styles indicate the contours obtained with three independent sets of background regions used for the corresponding background spectra extraction.

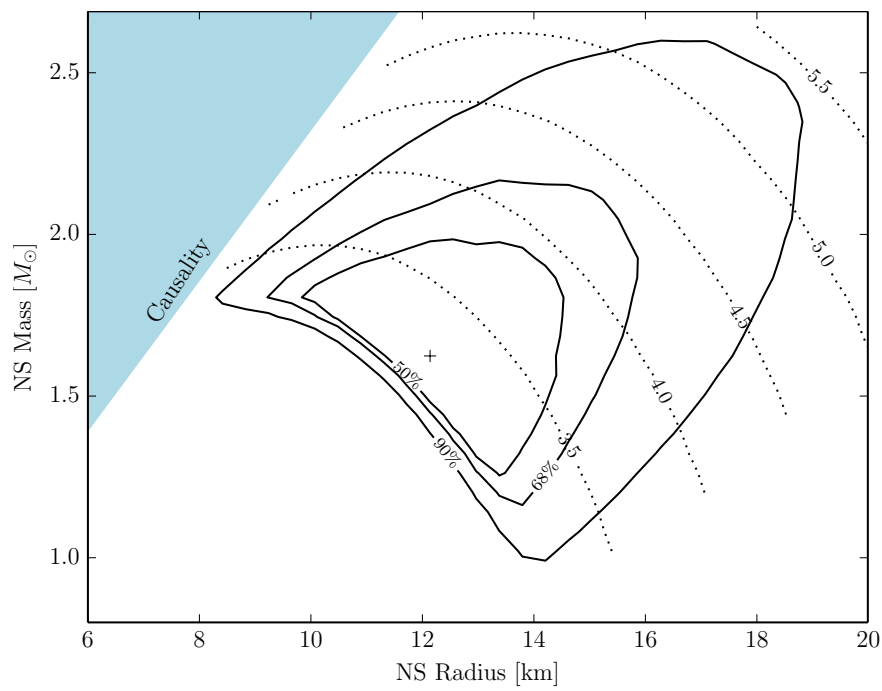

Fig. 5. Confidence regions similar to those shown in Fig. 3 but with the distance as a free fit parameter with a lower limit of $3.2 \mathrm{kpc}$. The dotted contours indicate best-fit distance in kpc computed for each point of the mass-radius grid.

that the displacements are by a factor of several smaller than the characteristic size of the contours. We thus conclude that the investigated systematics can be neglected at this stage.

As can be seen in Table 2, the spectral models with $d=3.2 \mathrm{kpc}$ and $d=4.5 \mathrm{kpc}$ provide the same fit quality. Nevertheless, the model is sensitive to the distance as seen in Fig. 5. The figure shows the $M-R$ contours with the distance being a free fit parameter with a lower limit of $3.2 \mathrm{kpc}$. The best-fit distances are computed for each point of the $M-R$ grid and are indicated with the dashed contours. Distances larger than $\sim 5.5 \mathrm{kpc}$ lead to high $\chi^{2}$ values and are formally excluded under the assumption that the emitting region is the entire stellar surface.

\subsection{Fit with hydrogen atmosphere models}

The combined XMM-Newton spectra of XMMU J1732 were also fitted using our hydrogen NS atmosphere models. The model

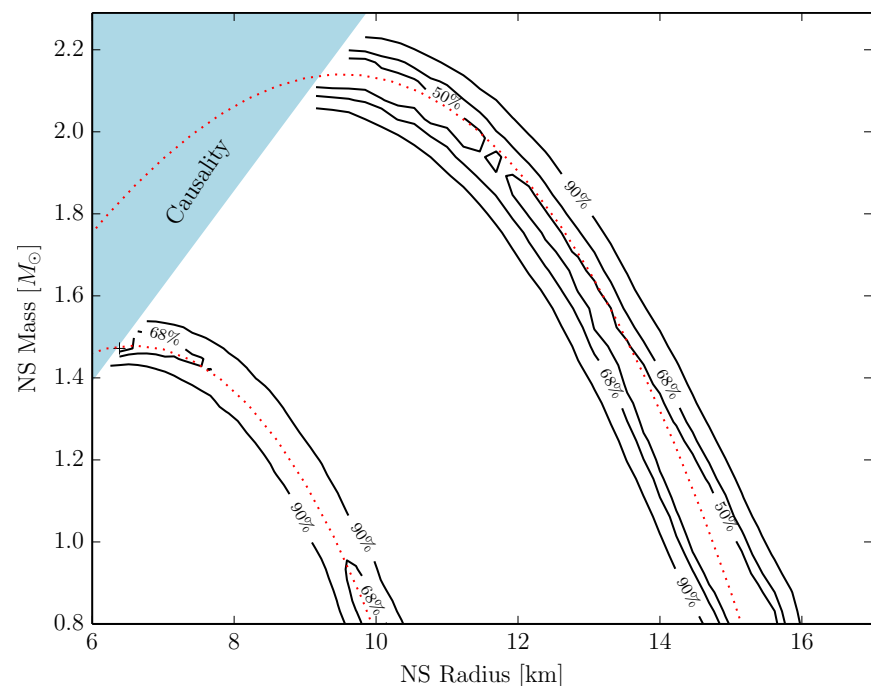

Fig. 6. Confidence regions similar to those in Fig. 3 but for the hydrogen atmosphere models and for the fixed distances of $7 \mathrm{kpc}$ (bottom left) and $10 \mathrm{kpc}$ (top right). The dotted curves indicate the lines of equal apparent radii: $R_{\infty}=11.4 \mathrm{~km}$ (bottom) and $R_{\infty}=16.5 \mathrm{~km}$ (top). The corresponding best-fit apparent temperatures are $2.76 \pm 0.02$ and $2.78 \pm$ $0.02 \mathrm{MK}$ for 7 and $10 \mathrm{kpc}$, respectively.

spectra are calculated for a range of effective temperatures of $0.5-10 \mathrm{MK}$ with a step size of $0.05 \mathrm{MK}$ and for the same set of nine surface gravity $\log$ arithms $\log g$ as for our carbon atmosphere models (Suleimanov et al. 2014). The models are computed using the LTE approximation with pressure ionization effects included using the occupation probability formalism (Hummer \& Mihalas 1988) as described by Hubeny et al. (1994). The method of modeling is described in detail by Suleimanov \& Werner (2007). We do not consider Compton scattering; all models are computed using coherent electron scattering alone. This approach is not correct for hot model atmospheres with $T>$ 3-5 MK (Suleimanov \& Werner 2007). Therefore, the hottest models have to be used with caution. It turns out, however, that the fits to our spectra yield lower temperatures. Similar to the carbon atmosphere models (previous section), the free parameters of the spectral model are the mass and radius of the star, the effective temperature $T$, the equivalent hydrogen column density, and the distance $d$.

As mentioned in Sect. 4.1, the fit quality in case of the hydrogen models is similar to the carbon model: $\chi_{\text {red }}^{2} \simeq 1.07$ for 895 d.o.f. (the corresponding $P$-value is $\sim 0.1$ ). The confidence contours in the $M-R$ plane resulting from the fit with the hydrogen models are presented in Fig. 6 for two fixed distances, 7 and $10 \mathrm{kpc}$. The distances were chosen for illustration only. For $d<7 \mathrm{kpc}$ the contours require masses and radii smaller than the canonical values usually assumed for an NS. The distances above $10 \mathrm{kpc}$ would imply an unrealistically high TeV luminosity of the SNR. Thus, under the assumption that the emission is uniformly produced by the entire stellar surface, a hydrogen atmosphere seems to be incompatible with the canonical masses and radii of an NS and with the available constraints on the source distance.

It is interesting to note that the contours are located along the respective lines of equal apparent radii indicated by the dotted curves. The model thus behaves similar to a blackbody function where the apparent radius is coupled to the normalization for a fixed distance and is therefore a fit parameter. The apparent temperatures $T_{\infty}=T /(1+z)$ measured with the hydrogen 


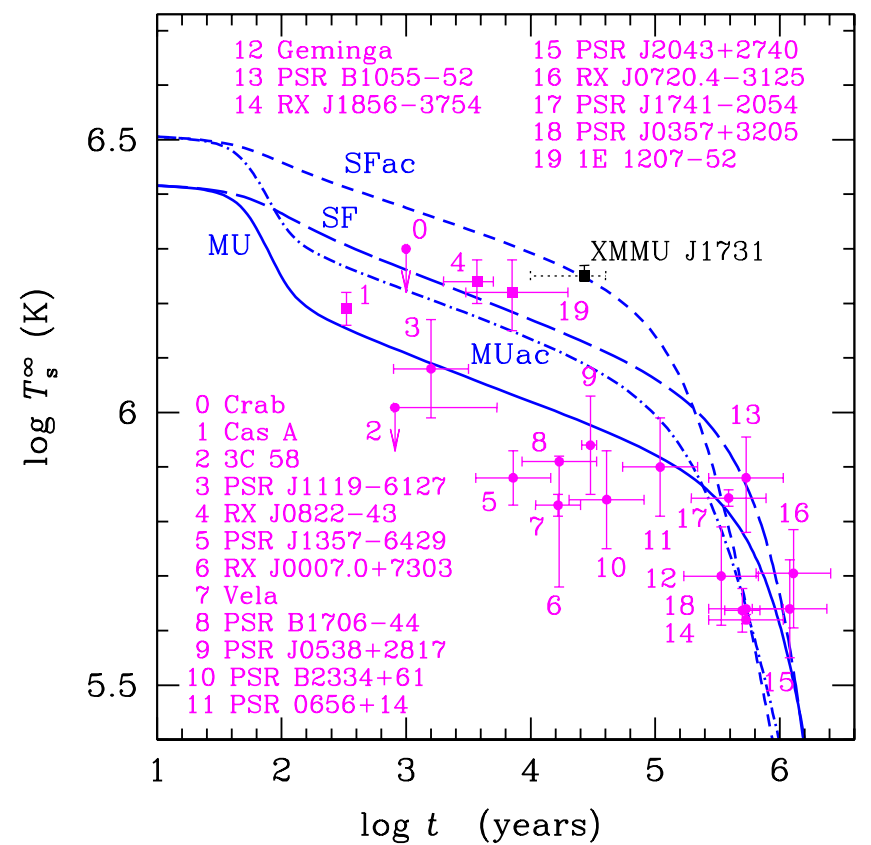

Fig. 7. Effective surface temperatures or upper limits for a number of cooling isolated NSs including XMMU J1732 versus their ages (data points) compared with four theoretical cooling curves for a $1.5 M_{\odot}$ star. The plot contains four CCOs with reliable temperature measurements (labeled with squares). For XMMU J1732, the dashed error bar indicates a conservative range of $10-40 \mathrm{kyr}$ adopted for the NS age. MU refers to a non-superfluid star with a heat blanket made of iron; SF corresponds to a star with strong proton superfluidity in the core and the same heat blanket; MUac and SFac refer, respectively, to the same models as MU and SF but with a fully carbon heat blanketing envelope (see text for details).

models are $2.76 \pm 0.02$ and $2.78 \pm 0.02 \mathrm{MK}$ for 7 and $10 \mathrm{kpc}$, respectively.

For completeness, we provide the best-fit absorption column density obtained with the hydrogen model, $n_{\mathrm{H}}=(1.91 \pm 0.03) \times$ $10^{22}$ atom $\mathrm{cm}^{-2}$. Since the confidence contours are not closed (Fig. 6), mass, radius, and effective temperature are formally unconstrained by the fit.

As mentioned in Suleimanov et al. (2014) and Paper I, a number of effects are not taken into account in our calculations of the carbon atmosphere models such as the influence of the magnetic field and a possible complex chemical composition different from pure carbon. The same applies to the hydrogen atmosphere models described above. These effects might influence the shape of the spectrum and thus the presented $M-R$ confidence contours. We believe, however, that our models are applicable for $B \lesssim 10^{8}-10^{9} \mathrm{G}$ as explained in Paper I. Since no pulsations are detected in XMMU J1732, no estimates of the surface $B$-field strength are available and our assumption of low magnetic field can be valid.

We also note that the values of the interstellar absorption column density obtained with both carbon and hydrogen models are within the range of the column densities measured in different parts of the remnant (Abramowski et al. 2011).

\section{An exceptionally hot cooling neutron star}

Since the apparent temperature of the CCO in HESS J1731-347 is well measured, it is interesting to investigate its status among other NSs of this family. Figure 7 shows a representative sample of cooling isolated NSs in a $T_{\mathrm{s}}^{\infty}-t$ diagram, where $t$ is the estimated/measured age of the objects and $T_{\mathrm{s}}^{\infty}$ is the apparent effective surface temperature (here we denote the surface temperature as $T_{\mathrm{S}}$ to distinguish it from the internal temperature of the star, $T_{\mathrm{i}}$ ). The data are the same as in Weisskopf et al. (2011) with an addition of three new sources, XMMUJ1732 (this work), PSR J1741-2054 (Karpova et al. 2014), and PSR J0357+3205 (Marelli et al. 2013; Kirichenko et al. 2014). We have also added 1E 1207.4-5209 (Zavlin et al. 1998).

The data displayed in Fig. 7 contain four CCOs which are marked by squares (to distinguish from other sources labeled by filled dots). Thermal emission of two CCOs, the NS in Cas A and XMMU J1732, can be interpreted as radiation from the entire star surface with realistic mass and radius using the carbon atmosphere models as explained in detail in Ho \& Heinke (2009), Paper I, and in this work. As for RX J0822-4300 (CCO in the SNR Puppis A) and 1E 1207.4-5209 (CCO in the SNR G296.5+10.0), their emission can be interpreted with hydrogen atmosphere models (Zavlin et al. 1998, 1999).

The indicated position of XMMU J1732 corresponds to $d=$ $3.2 \mathrm{kpc}$ and $t=27 \mathrm{kyr}$. The age was estimated by Tian et al. (2008) using the Sedov solution for the SNR assuming a distance of $3.2 \mathrm{kpc}$. The error bars on $T_{\mathrm{s}}^{\infty}$ are enlarged by a factor of two for a better visualization. The uncertainties of the age estimate are not well understood. We adopt a conservative range of 10-40 kyr. For larger distances, the age must be larger. One can see that the CCO appears exceptionally hot for the assumed age, i.e., it must have been cooling very slowly posing a challenge for the cooling theory.

According to the theory, middle-aged isolated NSs (as those shown in Fig. 7) have already passed the initial cooling stage of internal thermal relaxation - the first 10-100 yrs depending on the NS model (e.g., Yakovlev \& Pethick 2004; Page et al. 2009). Their internal layers are almost isothermal. A strong temperature gradient still persists in the outer heat blanketing envelopes (under the atmosphere, a few ten meters thick). Such stars are mostly cooling "from inside" (mostly from their superdense cores) via neutrino emission. The photon thermal luminosity only becomes significant in cooling at $t \gtrsim 10^{5} \mathrm{yr}$. The decay of the internal temperature $T_{i}$ is determined by the neutrino cooling function

$\ell\left(T_{i}\right)=L_{\nu}\left(T_{i}\right) / C\left(T_{i}\right)$,

where $L_{v}\left(T_{\mathrm{i}}\right)$ and $C\left(T_{\mathrm{i}}\right)$ are the neutrino luminosity and the heat capacity of the star, respectively. Accounting for general relativity, $T_{\mathrm{i}}, L_{v}$, and $C$ have to be redshifted in the cooling equations. Thus, the internal cooling is regulated by the neutrino emission and the heat capacity. The effective surface temperature $T_{\mathrm{s}}$ simply adjusts itself to the internal temperature $T_{\mathrm{i}}$. The adjustment is taken into account using calculated $T_{\mathrm{s}}\left(T_{\mathrm{i}}\right)$-relations. These relations are determined by the thermal conductivity of the heat blanketing envelopes which is affected by their chemical composition.

The four theoretical cooling curves $T_{\mathrm{s}}^{\infty}(t)$ presented in Fig. 7 are calculated using a generally relativistic cooling code (Gnedin et al. 2001). The curves are aimed at explaining the hottest cooling NSs. Colder NSs are commonly treated as sufficiently massive stars with higher neutrino emission from the core. In Fig. 7, we use an NS model with a nucleon core (the modification of the APR EOS of superdense matter used in Weisskopf et al. 2011). The NS mass is $M=1.5 M_{\odot}$ and radius $R=12.03 \mathrm{~km}$. The cooling curves are, however, known to be fairly independent of EOS, $M$, and $R$ for typical theoretical neutron stars $\left(M \lesssim 1.8 M_{\odot}\right.$, $R \sim 10-13 \mathrm{~km}$ ) where the direct Urca process does not operate (e.g., Yakovlev \& Pethick 2004). 
The neutrino emission from the core of the given star can be produced by the modified Urca process (MU) and somewhat weaker processes of nucleon-nucleon collisions (but no powerful direct Urca process). Neutron superfluidity in the core due to triplet-state pairing is neglected, proton superfluidity due to single-state pairing is varied.

Further we assume a blanketing envelope made of carbon with a mass of $\Delta M$ (Yakovlev et al. 2011). For $\Delta M \lesssim 10^{-14} M_{\odot}$, the amount of carbon is too small to affect the cooling. At $\Delta M \sim 10^{-8} M_{\odot}$ the amount of carbon is maximal. For formally higher $\Delta M$, carbon would transform into heavier elements at the bottom of the envelope because of beta captures and pycnonuclear reactions. We neglect the effects of the magnetic field on the cooling which is a good approximation as long as $B \lesssim 10^{12} \mathrm{G}$.

The solid cooling curve (MU) corresponds to a nonsuperfluid star with an ordinary (iron) heat blanketing envelope. This is a basic cooling curve (cooling through the modified Urca process non-affected by superfluidity, the so-called standard neutrino candle, Yakovlev et al. 2011). It is consistent with observations of some NSs, but cannot explain XMMU J1732 which is substantially hotter. One can obtain a hotter star by switching on proton superfluidity and replacing iron with carbon in the heat blanketing envelope. The long-dashed cooling curve (SF) corresponds to strong proton superfluidity in the NS core (with the critical temperature over the core $T_{\mathrm{cp}}(\rho) \gtrsim 3 \times 10^{9} \mathrm{~K}$, where $\rho$ is the density) and an iron heat blanket. The exact $T_{\mathrm{cp}}(\rho)$ profile in this limit is not important. Such superfluidity almost completely switches off the proton heat capacity (reducing the total heat capacity by $\sim 25 \%$, Page 1993) and all neutrino processes involving protons, first of all the modified Urca process. The neutrino luminosity from the core is determined by neutron-neutron collisions and is about two orders of magnitude weaker than the modified Urca. This greatly reduces the neutrino luminosity function (1), slows down the internal neutrino cooling, and makes the star hotter (at $t \gtrsim 100 \mathrm{yr}$, after the internal thermal relaxation for the given NS model). However, it is still insufficient to explain the temperature of XMMU J1732 at the assumed age.

One might think that one can additionally slow down the cooling by assuming strong superfluidity of neutrons in the core. It will indeed block neutrino emission due to neutron-neutron collisions leaving a much weaker neutrino emission caused by electron-electron collisions. It will, however, also block the neutron heat capacity in the core (the main source of heat capacity there, Page 1993) producing a neutron star with very low neutrino luminosity and heat capacity in the core. Its cooling will be greatly affected by the microphysics of the crust and will not be much slower than that without neutron superfluidity (see, e.g., Yakovlev \& Pethick 2004).

The dotted-dashed cooling curve (MUac) in Fig. 7 refers to a non-superfluid star but with a fully carbon heat blanketing envelope $\left(\Delta M / M=10^{-8}\right)$. Internally, this star cools down as the standard candle (through the modified Urca process) but the heat blanket is now made of lighter, more heat transparent carbon. This increases the surface temperature (Yakovlev et al. 2011). However, such an increase is still insufficient to explain XMMU J1732.

Finally, the short-dashed curve in Fig. 7 corresponds to strong proton superfluidity and a fully carbon heat blanket. Now we have used all cooling regulators to slow down the cooling and to obtain an exceptionally hot NS. This scenario is formally consistent with the data of XMMU J1732. Therefore, with the assumed age and distance, the data formally require both very low neutrino cooling rate (low $\ell$ ) and a fully carbon heat blanket.

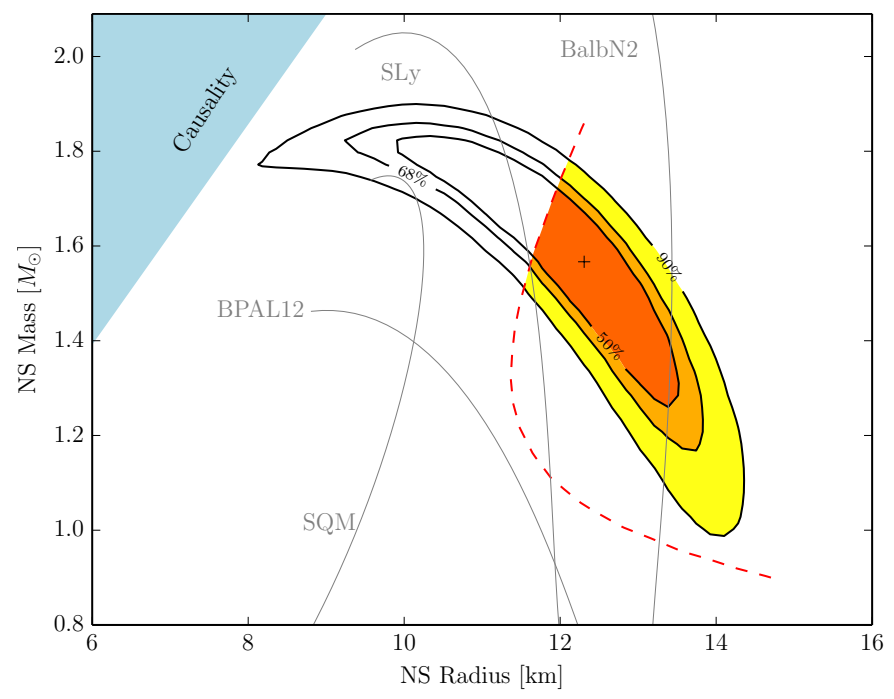

Fig. 8. Confidence regions similar to those in Fig. 3 for a distance of $3.2 \mathrm{kpc}$ where the area on the right side of the red dashed curve indicates the region allowed by the cooling theory assuming the NS age of $27 \mathrm{kyr}$ (see text for details).

Such a slowly cooling NS would be a remarkable object. The neutrino emission from its core is so slow that the neutrino emission from the crust starts to affect the cooling.

We repeat that the above considerations assume $t=27 \mathrm{kyr}$ and $d=3.2 \mathrm{kpc}$. For a distance of $4.5 \mathrm{kpc}$, the Sedov solution would yield $t \simeq 40 \mathrm{kyr}$ and the problems related to the NS cooling would become even more severe. The temperatures derived using the blackbody and hydrogen models, which are not well justified (Sects. 4.1 and 4.3), turn out to be much higher than for the carbon model so that the NS would appear even hotter. If, however, XMMU J1732 is younger, e.g., $t \sim 10 \mathrm{kyr}$, the discrepancy with the cooling theory is reduced. However, even in that case we would need both strong proton superfluidity and a lot of carbon in the heat blanket, although superfluidity could be somewhat weaker (lower $T_{\mathrm{cp}}$ ) and/or the amount of carbon could be lower (smaller $\Delta M$ ).

The case of $t=27 \mathrm{kyr}$ requires extreme parameters of both cooling regulators (strong proton superfluidity and maximum amount of carbon). As discussed above, the theoretical cooling curves are almost independent of EOS, $M$ and $R$ in a broad range of $M$ and $R$. However, they do depend on $M$ and $R$ outside that range. In case of the observed $T_{\mathrm{s}}^{\infty}$, they slightly depend on $M$ and $R$. Therefore, the explanation of the observed $T_{\mathrm{s}}^{\infty}$ can fail at certain $M$ and $R$ where the theoretical cooling curve goes below the inferred $T_{\mathrm{s}}^{\infty}$ even at the extreme parameters of the cooling regulators. These values of $M$ and $R$ can be treated as forbidden by the cooling theory which gives additional constraints on $M$ and $R$. A precise derivation of such constraints is beyond the scope of this work and will be presented elsewhere. In Fig. 8, we present our preliminary constraints for $t=27 \mathrm{kyr}$. They are obtained using the rescaling relations which allows us to calculate $T_{\mathrm{s}}^{\infty}$ for a slowly cooling middle-aged NS in a wide range of $t, M, R$, and EOSs. The relations are then calibrated with respect to theoretical cooling curves in Fig. 7. The allowed region in Fig. 8 is on the right side of the red dashed curve. One can see that under the stated assumptions the cooling theory mostly eliminates the range of small $R \lesssim 12 \mathrm{~km}$.

As an alternative explanation of the exceptionally high $T_{\mathrm{s}}^{\infty}$, additional heating mechanisms inside the star can be invoked (e.g., Yakovlev \& Pethick 2004; Page et al. 2006). In our case, 
these mechanisms are, however, not easy to justify because they are known to be mostly efficient in old and cold NSs whereas XMMU J1732 is very hot.

\section{Discussion and conclusions}

The new observations continue the long-term monitoring of XMMU J1732 and confirm the lack of long-term variations in the source flux or spectrum. Although the lack of any long-term variability is considered to be a common property of CCOs, a secular decrease of the NS effective temperature ("cooling") was reported for the CCO in Cas A by Heinke \& Ho (2010). The cooling is, however, questioned in the recent work of Posselt et al. (2013) where the authors analyzed two Chandra observations obtained in a pileup-free observing mode.

The factor of five increase in the exposure time compared to the previously available XMM-Newton observations of XMMU J1732 reported in Paper I substantially improves constraints on the spectral continuum of the source. For the first time, the pure blackbody spectral function is clearly rejected based solely on the fit quality rather than on the best-fit values of the model parameters (as discussed, e.g., in Paper I). The carbon or hydrogen atmosphere models are clearly preferred by the fit.

The new observations did not reveal any pulsations with pulsed fraction above $\sim 7-8 \%$ and a period longer than $\sim 0.15 \mathrm{~s}$. In combination with the absence of pulsations at higher frequencies reported in Paper I with a similar upper limit on the pulsed fraction, this result supports the hypothesis that the emitting area is identical or close to the entire stellar surface. We maintain this hypothesis in the following discussion. We admit, however, that the available upper limits on the pulsed fraction of the order of $\sim 0.1$ is not very stringent. Two of the three known pulsating CCOs, RX J0822.0-4300 and 1E 1207.4-5209, exhibit pulsed fractions of only 11 and 9\%, respectively (Gotthelf et al. 2013, and references therein). One thus cannot completely reject the possibility that the emitting area is somewhat smaller than the stellar radius. Stronger upper limits on the pulsed fraction are necessary.

Under the assumptions of the two most probable distances, 3.2 or $4.5 \mathrm{kpc}$, the carbon atmosphere models lead to relatively compact confidence regions in the NS mass-radius plane (Fig. 3). The contours corresponding to $d=3.2 \mathrm{kpc}$ are compatible with the most commonly used nuclear EOSs and with the canonical NS mass and radius of $\sim 1.4 M_{\odot}$ and $\sim 12 \mathrm{~km}$, respectively. The contours corresponding to $d=4.5 \mathrm{kpc}$ require mass and radius which appear to be uncomfortably large for an NS. The fit with the carbon atmosphere models thus supports the possibility of the source located in the Scutum-Crux spiral arm at $\sim 3 \mathrm{kpc}$ over the location in the Norma arm at $\sim 4.5 \mathrm{kpc}$. Distances substantially larger than $\sim 5-6 \mathrm{kpc}$ are excluded solely by the spectral fit under the stated assumptions (Fig. 5). The sensitivity to the distance is a promising feature of the carbon model which can be utilized in the the spectral analyses of isolated lowmagnetized NSs in the future.

Interesting results concerning the applicability of our carbon atmosphere models has recently been presented by Bogdanov (2014) who investigated the CCO in the SNR Kes 79. Contrary to all other CCOs, this object exhibits an extremely high pulsed fraction of $\sim 64 \%$. The author demonstrates that the X-ray spectrum of the source can be well fitted with the model of a pure carbon atmosphere uniformly covering the entire surface of a star with a radius of $\sim 14 \mathrm{~km}$ at the adopted distance of $7 \mathrm{kpc}$ although such a uniformity apparently contradicts the observed high pulsed fraction. Therefore, further searches for pulsations or the improvements of the upper limits on the pulsed fraction for the CCOs in Cas A and in HESS J1731-347 are of key importance for the chemical composition of the NS atmosphere and the derived parameters of the NSs.

The hydrogen atmosphere models are applied to XMMUJ1732 for the first time. Contrary to carbon, they turn out to be not very sensitive to the distance. The mass-radius contours obtained for a fixed distance are not closed. For distances below $\sim 7 \mathrm{kpc}$ the contours indicate the masses and radii which are substantially lower than those usually assumed for NSs. However, they are compatible with a possibility of a strange star covered by ordinary matter (hydrogen). Such hypothetic stars built entirely or predominantly of self-bound quark matter may possess masses and radii much smaller than those of canonical NSs (e.g., Haensel et al. 2007). Besides this possibility and under the assumption that the emitting area is the entire star surface, the hydrogen atmosphere model seems to be incompatible with the available distance estimates of $\sim 3$ or $\sim 4.5 \mathrm{kpc}$.

Based on the presented analysis, a low-magnetized neutron star located in the Scutum-Crux spiral arm and covered by a uniformly emitting carbon atmosphere seems to be the most plausible hypothesis for the CCO in HESS J1731-347.

Recently, Fukuda et al. (2014) argued that the remnant HESS J1731-347 should be associated with the $3 \mathrm{kpc}$ expanding arm based on their morphological studies of the ${ }^{12} \mathrm{CO}$ and HI observations. The authors argued that the distance to the arm in the direction of the remnant is in the range 5.2-6.1 kpc. Such a distance is formally still compatible with the carbon atmosphere models (Fig. 5) but requires the mass and radius of the compact star even higher than for $4.5 \mathrm{kpc}$. On the other hand, in case of our hydrogen atmosphere models, the suggested 5.2-6.1 kpc would lead to unrealistically low NS masses and radii (Fig. 6). The distance suggested by Fukuda et al. (2014) is thus not favored by our studies. This possibility, however, cannot be rejected at this stage.

According to the current cooling theories, the $\mathrm{CCO}$ in HESS J1731-347 can be considered as an isolated cooling NS which must have been cooling down very slowly. For a reasonable assumption on the source age of $\sim 10-40 \mathrm{kyr}$, it is the hottest cooling NS observed so far. It must have a very low neutrino luminosity and an unusually heat-transparent blanketing envelope. The required low neutrino luminosity can be realized in a star with strong proton superfluidity in the core (where neutrino emission is produced by neutron-neutron collisions). High heat transparency can be provided by the presence of sufficient amount of carbon in the heat blanketing envelope. For a star's age of $27 \mathrm{kyr}$, the heat blanket should contain the maximum amount of carbon, $\Delta M \sim 10^{-8} M_{\odot}$. For an age of $\sim 10 \mathrm{kyr}$, proton superfluidity can be weaker and/or $\Delta M$ can be smaller. In any case, the star should have a rather large amount of carbon in the surface layers. We demonstrated that the cooling theory has a potential to put further constraints on the mass and radius of XMMU J1732. It also seems to disfavor larger distances to the source, above $\sim 4-5 \mathrm{kpc}$, as they lead to a larger age of the NS.

Generally, our analysis demonstrates that CCOs are very promising objects for the measurements of NS masses and radii and, thus, for probing the EOS of superdense matter. The increase in the total exposure time led to a significant reduction of the allowed region in the mass-radius plane. Some constraints on mass and radius can also be given with only a lower limit on the distance (Fig. 5). The obtained best-fit NS radius of $12.4 \mathrm{~km}$ (for the preferred distance of $3.2 \mathrm{kpc}$ ) turns out to be very close to the preferable NS radii of $11-13 \mathrm{~km}$ obtained with the current 
theoretical modeling of EOSs, the experimental data from heavy ion collisions, and the observations of some X-ray bursting NSs and the NSs in quiescent states of soft X-ray transients (Steiner et al. 2013; Lattimer \& Steiner 2014).

As the future improvement of the observational data will further reduce the statistical errors on mass and radius, the uncertainties in the theoretical modeling might become the dominant source of errors. In this respect, we note the following physical processes not yet taken into account in our carbon NS atmosphere models which might affect the shape of the emergent spectra: (i) deviations of carbon ion number densities from LTE values (see, e.g., Rauch et al. 2008); (ii) non-coherent electron scattering (Compton effect); and (iii) influence of the magnetic field $B \sim 10^{10}-10^{11} \mathrm{G}$.

Acknowledgements. V.S. acknowledges the support by the German Research Foundation (DFG) grant SFB/Transregio 7 "Gravitational Wave Astronomy" and Russian Foundation for Basic Research (grant 12-02-97006-r-povolzhe-a). D.Y. acknowledges partial support by the Russian Foundation for Basic Research (grants No. 14-02-00868-a and 13-02-12017-ofi-M) and by the State Program "Leading Scientific Schools of RF" (grant NSh 294.2014.2).

\section{References}

Abramowski, A., Acero, F., Aharonian, F., et al. 2011, A\&A, 531, A81 Acero, F., Pühlhofer, G., Klochkov, D., et al. 2009, in Proc. of the 31th ICRC 2009, Lodz [arXiv: 0907.0642]

Bamba, A., Pühlhofer, G., Acero, F., et al. 2012, ApJ, 756, 149

Bogdanov, S. 2014, ApJ, 790, 94

de Jager, O. C., Raubenheimer, B. C., \& Swanepoel, J. W. H. 1989, in Data Analysis in Astronomy, eds. V. di Gesu, L. Scarsi, P. Crane, et al., 21

Fukuda, T., Yoshiike, S., Sano, H., et al. 2014, ApJ, 788, 94

Gnedin, O. Y., Yakovlev, D. G., \& Potekhin, A. Y. 2001, MNRAS, 324, 725

Gotthelf, E. V., Halpern, J. P., \& Alford, J. 2013, ApJ, 765, 58

Haensel, P., Potekhin, A. Y., \& Yakovlev, D. G. 2007, Astrophys. Space Sci. Lib., 326, Neutron Stars 1: Equation of State and Structure

Halpern, J. P., \& Gotthelf, E. V. 2010a, ApJ, 725, 1384
Halpern, J. P., \& Gotthelf, E. V. 2010b, ApJ, 709, 436 Halpern, J. P., \& Gotthelf, E. V. 2010c, ApJ, 710, 941 Heinke, C. O., \& Ho, W. C. G. 2010, ApJ, 719, L167

Ho, W. C. G., \& Heinke, C. O. 2009, Nature, 462, 71

Hou, L. G., Han, J. L., \& Shi, W. B. 2009, A\&A, 499, 473

Hubeny, I., Hummer, D. G., \& Lanz, T. 1994, A\&A, 282, 151

Hummer, D. G., \& Mihalas, D. 1988, ApJ, 331, 794

Jansen, F., Lumb, D., Altieri, B., et al. 2001, A\&A, 365, L1

Karpova, A., Danilenko, A., Shibanov, Y., Shternin, P., \& Zyuzin, D. 2014, ApJ, 789, 97

Kirichenko, A., Danilenko, A., Shibanov, Y., et al. 2014, A\&A, 564, A81

Klochkov, D., Pühlhofer, G., Suleimanov, V., et al. 2013, A\&A, 556, A41

Lattimer, J. M., \& Prakash, M. 2007, Phys. Rep., 442, 109

Lattimer, J. M., \& Steiner, A. W. 2014, ApJ, 784, 123

Marelli, M., De Luca, A., Salvetti, D., et al. 2013, ApJ, 765, 36

Page, D. 1993, in Nuclear Physics in the Universe, eds. M. W. Guidry, \& M. R. Strayer, 151

Page, D., Geppert, U., \& Weber, F. 2006, Nuclear Physics A, 777, 497

Page, D., Lattimer, J. M., Prakash, M., \& Steiner, A. W. 2009, ApJ, 707, 1131

Pavlov, G. G., \& Luna, G. J. M. 2009, ApJ, 703, 910

Pavlov, G. G., Sanwal, D., Garmire, G. P., \& Zavlin, V. E. 2002, in Neutron Stars in Supernova Remnants, eds. P. O. Slane, \& B. M. Gaensler, ASP Conf. Ser., 271, 247

Pavlov, G. G., Sanwal, D., \& Teter, M. A. 2004, in Young Neutron Stars and Their Environments, eds. F. Camilo, \& B. M. Gaensler, IAU Symp., 218, 239 Posselt, B., Pavlov, G. G., Suleimanov, V., \& Kargaltsev, O. 2013, ApJ, 779, 186 Protheroe, R. J. 1987, Proc. Astron. Soc. Aust., 7, 167

Rauch, T., Suleimanov, V., \& Werner, K. 2008, A\&A, 490, 1127

Steiner, A. W., Lattimer, J. M., \& Brown, E. F. 2013, ApJ, 765, L5

Strüder, L., Briel, U., Dennerl, K., et al. 2001, A\&A, 365, L18

Suleimanov, V., \& Werner, K. 2007, A\&A, 466, 661

Suleimanov, V. F., Klochkov, D., Pavlov, G. G., \& Werner, K. 2014, ApJS, 210, 13

Tian, W. W., Leahy, D. A., Haverkorn, M., \& Jiang, B. 2008, ApJ, 679, L85

Tian, W. W., Li, Z., Leahy, D. A., et al. 2010, ApJ, 712, 790

Turner, M. J. L., Abbey, A., Arnaud, M., et al. 2001, A\&A, 365, L27

Weisskopf, M. C., Tennant, A. F., Yakovlev, D. G., et al. 2011, ApJ, 743, 139

Yakovlev, D. G., \& Pethick, C. J. 2004, ARA\&A, 42, 169

Yakovlev, D. G., Ho, W. C. G., Shternin, P. S., Heinke, C. O., \& Potekhin, A. Y. 2011, MNRAS, 411, 1977

Zavlin, V. E., Pavlov, G. G., \& Trumper, J. 1998, A\&A, 331, 821

Zavlin, V. E., Trümper, J., \& Pavlov, G. G. 1999, ApJ, 525, 959 